\title{
Calidad de vida en pacientes con demencia tipo Alzheimer
}

\section{Quality of life in patients with Alzheimer's dementia}

Cañas Lucendo Manuel

Universidad de Chimborazo, Ecuador

Perea Bartolomé María Victoria

Universidad de Salamanca, España

Ladera Fernández Valentina

Universidad de Salamanca, España

García Ricardo Pérez

Universidad de Salamanca, España

Sáez Enrique Escuela

Centro de Referencia estatal de atención a personas con enfermedad de alzheimer y otras demencias de Salamanca, España

Autor para correspondencia: mcanas@unach.edu.ec

Fecha de recepción: 23 de enero 2018 - Fecha de aceptación: 26 de febrero de 2018

Resumen: La Demencia tipo Alzheimer es una enfermedad neurodegenerativa que produce síntomas cognitivos y conductuales que afectan la calidad de vida. La calidad de vida es la percepción que un individuo tiene sobre su propia salud. Se realizó una investigación cuya muestra del estudio estaba constituida por 50 pacientes con Demencia tipo Alzheimer y 60 Sujetos sin deterioro cognitivo. Se aplicó la Escala de calidad de vida en la Enfermedad de Alzheimer- QOLAD. Los resultados demuestran que los pacientes con Demencia tipo Alzheimer tienen una menor calidad de vida global auto percibida que los sujetos sin deterioro cognitivo.

Palabras claves: demencia tipo Alzheimer; calidad de vida

Abstract: Alzheimer's Dementia is a neurodegenerative disease that produces cognitive and behavioral symptoms that affect the quality of life. The quality of life is the perception that an individual has about their own health. An investigation was carried out whose sample of the study consisted of 50 patients with Alzheimer's Dementia and 60 Subjects without cognitive impairment. The Quality of Life Scale in Alzheimer's Disease- QOL-AD was applied. The results show that patients with Alzheimer's Dementia have a lower overall self-perceived quality of life than subjects without cognitive impairment.

Key words: Alzheimer's dementia; quality of life 


\section{Introducción}

La Calidad de Vida (CV) ha sido definida por la Organización Mundial de la Salud (OMS) como: "la percepción que un individuo tiene de su lugar en la existencia, en el contexto de la cultura y del sistema de valores en los que vive y en relación con sus objetivos, expectativas, normas e inquietudes. Se trata de un concepto influido por la salud física del sujeto, su estado psicológico, su nivel de independencia, sus relaciones sociales, así como su relación con los elementos esenciales de su entorno" (WHO, 1994).

La Demencia tipo Alzheimer (DTA) es un síndrome neurodegenerativo caracterizado por el deterioro progresivo e irreversible de múltiples funciones cognitivas junto con alteraciones graves de la conducta y funcionales que repercuten sobre la CV (Perea, 2005). Los pacientes con DTA presentan un severo deterioro cognitivo, junto con la presencia de trastornos neuroconductuales y alteraciones funcionales que repercuten sobre su $\mathrm{CV}$.

A pesar de que los nuevos modelos de atención centrada en la persona consideran que hay que tener en cuenta la opinión de la persona con el objetivo de mantener la individualidad y la independencia de la persona, la mayoría de estudios de investigación analizan la percepción de la CV desde el punto de vista del cuidador principal (Nogueira et al, 2015). Recientemente otros enfoques, tienen en cuenta la comparación entre la percepción de la CV entre el paciente y su cuidador principal (Bosboom, Alfonso \& Almeida, 2013; Conde-Sala et al., 2014), aunque se constatan la ausencia de estudios que analicen la CV desde el punto de vista o autopercepción del paciente con DTA. Una posible explicación es mantenida por algunos investigadores que han planteado que los pacientes con DTA debido a su deterioro cognitivo, no son capaces de informar de forma fiable y valida sobre su percepción de CV (Gómez-Gallego, 2012).

Para valorar la CV en la DTA, se han utilizado cuestionarios y escalas como la Escala de Calidad de Vida en la Enfermedad de Alzheimer (QOL-AD) (Logsdon, Gibbons, McCurry y Teri, 1999) que han demostrado que la CV no empeora conforme disminuye el nivel de deterioro cognitivo en la demencia. Estos datos sugieren por tanto que no existe una relación entre la CV y la presencia de deterioro cognitivo. A pesar de la creencia generalizada de que la CV no se agrava o empeora conforme evoluciona la demencia y el deterioro cognitivo, otros estudios han constatado que la mejoría en la cognición y el estado de ánimo puede dar lugar a un aumento significativo de la CV (Hoe et al., 2009).

Estos hallazgos cuestionan la relación existente entre la CV y el deterioro cognitivo, por lo que justifican que sea necesario investigar más con el objetivo de determinar si existe diferencias entre la percepción de la CV en pacientes con DTA y SSDC.

Por esta razón, el objetivo del presente trabajo de investigación consiste en la medición de la CV basada en la autopercepción que tiene el paciente con DTA, en comparación con un grupo de Sujetos sin deterioro cognitivo (SSDC) con la finalidad de demostrar la hipótesis de que a mayor afectación del deterioro cognitivo menor $\mathrm{CV}$ auto percibida en pacientes con DTA. 
Las implicaciones del estudio permitirán mejorar el conocimiento sobre si la CV se ve afectada por el deterioro cognitivo, lo que podría generar un aumento de estudios de investigación para determinar que funciones cognitivas se ven más relacionadas con la CV.

\section{Métodos}

Diseño:

Observacional transversal.

\section{Población y Muestra:} de estudio:

Selección mediante un muestreo no probalístico. La muestra está dividida en dos grupos

$\checkmark$ Grupo $\mathrm{n}^{\mathrm{o}}$ 1: Pacientes con diagnóstico de DTA.

$\checkmark$ Grupo $\mathrm{n}^{\circ} 2$ : SSDC con características sociodemográficas similares al grupo 1.

\section{Criterios de inclusión:}

Los criterios para formar parte del grupo 1 son los siguientes:

$\checkmark$ Diagnóstico clínico de demencia realizado por un médico especialista en Neurología.

$\checkmark$ Cumplir con los criterios diagnósticos de demencia establecidos en el DSM-IV-TR (APA, 2002).

$\checkmark$ Cumplir con los criterios diagnósticos de Enfermedad de Alzheimer probable establecidos por el NINCDS-ADRDA (McKhann et al., 1984).

$\checkmark$ Deterioro cognitivo general; Obtener una puntuación < de 24 en el Examen Cognoscitivo Mini Mental adaptación española- MMSE (Lobo, Saz, Marcos y ZARADEMP, 2002).

$\checkmark$ No presentar en su historia clínica alteraciones neurológicas, neuroquirúrgicas, neuropsicológicas y/o psicopatológicas clínicamente demostrables.

\section{Instrumento:}

Escala de calidad de vida en la Enfermedad de Alzheimer- QOL-AD, versión del paciente (Logsdon et al., 1999). Es una escala breve de valoración de la calidad de vida en pacientes con DTA, cuyo índice de consistencia interna reveló valores aceptablemente altos $(\alpha=84)$ (Logsdon et al., 1999).

\section{Procedimiento:}

Una vez obtenida la autorización por parte del Centro de Referencia Estatal de Atención a Personas con Enfermedad de Alzheimer y otras Demencias (IMSERSO) de Salamanca (España), se realizó una revisión de los historiales clínicos teniendo en cuenta la evaluación neurológica, con el objetivo de realizar una confirmación del diagnóstico de DTA. Posteriormente se seleccionó a los participantes que cumplían con los criterios de inclusión anteriormente 
mencionados. Se les aplicó el consentimiento informado para participar en la investigación y se comenzó la aplicación de la Escala de evaluación de la CV.

Análisis de los datos:

Para el estudio estadístico se utilizó el software IBM SPSS Stadistic, versión 22, mediante el cual se han empleado una serie de técnicas y test estadísticos como el empleo de tablas de frecuencias, porcentajes y datos descriptivos como media, mediana, desviación estándar.

\section{Consideraciones éticas:}

El proyecto de investigación fue aprobado por el área de Formación, Evaluación, Investigación del Centro de Referencia Estatal de Atención a Personas con Enfermedad de Alzheimer y otras Demencias de Salamanca (España) y posteriormente se aplicó el consentimiento informado, garantizando la confidencialidad de los datos y haciendo mención expresa de que la investigación no entrañaba ningún tipo de peligro potencial.

\section{Resultados}

En la Tabla 1 se observan las características sociodemográficas de la muestra, formada por 110 participantes, 83 mujeres $(75,5 \%)$ y 23 hombres $(24,5 \%)$. La edad media de los sujetos es de 74,93 (DS=8,97), con un rango desde los 55- 90 años de edad y mediana de 77 años de edad. Las edades medias de hombres $(74.41+8.92)$ y de mujeres $(75.10+9.04)$ son muy similares, así como sus rangos (56-88 y 56-90, respectivamente) de manera que no existen diferencias significativas entre ellos con $\mathrm{P}>.05$ (Student: $\mathrm{t}=-0.34 ; 108 \mathrm{gl} ; \mathrm{P}=.731$ ). Sin embargo, sí se han encontrado diferencias en la variable "edad" $(\mathrm{t}=5.71 ; \mathrm{P}<.001)$, de forma que los SSDC son más jóvenes (71 años) que los casos con DTA (79.6); diferencia que se estima al 95\% de confianza entre 5.6 y 11.64 años.

Tabla 1: Análisis descriptivo y comparativo. Características sociodemográficas de la muestra total y por grupo

\begin{tabular}{|c|c|c|c|c|c|c|c|}
\hline Variable & Categoría & $\begin{array}{l}\text { Muestra total } \\
(\mathrm{N}=110)\end{array}$ & DTA $(\mathbf{N}=50)$ & $\operatorname{SSDC}(N=60)$ & Estadístico & Gl & $\mathbf{P}$ \\
\hline & Mujer & $75.5 \%(83)$ & $74.0 \%(37)$ & $76.7 \%$ & & & \\
\hline Género & Hombre & $24.5 \%(27)$ & $26.0 \%(13)$ & $23,3 \%(14)$ & Chi2 $=0.11$ & 1 & $.746 \mathrm{NS}$ \\
\hline Edad & & $74.93+8.98$ & $79.64+7.33$ & $71.00+8.35$ & $\mathrm{t}=5.71$ & 108 & $.000 * *$ \\
\hline Estado Civil & $\begin{array}{l}\text { Soltero } \\
\text { Casado } \\
\text { Viudo }\end{array}$ & $\begin{array}{l}74.93+8.98 \\
2,7 \%(3) \\
59.1 \%(65) \\
38.2 \%(42)\end{array}$ & $\begin{array}{l}52.0 \%(26) \\
48.0 \%(24)\end{array}$ & $\begin{array}{l}5.0 \%(3) \\
65.0 \%(39) \\
30.0 \%(18)\end{array}$ & Chi $2=5.59$ & 2 & $.061 \mathrm{NS}$ \\
\hline Nivel Educativo & Estud.Primarios & $77.3 \%(85)$ & $76.0 \%(38)$ & $78.3 \%(47)$ & Chi $2=5.56$ & 4 & $.059 \mathrm{NS}$ \\
\hline
\end{tabular}




\begin{tabular}{|c|c|c|c|c|c|c|c|}
\hline & $\begin{array}{l}\text { Estud. } \\
\text { Secundarios }\end{array}$ & $16.4 \%(18)$ & $12.0 \%(6)$ & $20.0 \%(12)$ & & & \\
\hline & Estud. Superiores & $6.4 \%(7)$ & $12.0 \%(6)$ & $1.7 \%(1)$ & & & \\
\hline $\begin{array}{l}\text { Años } \\
\text { Escolarización }\end{array}$ & & $7.82+3.35$ & $7.92+3.83$ & $7.73+2.91$ & $\mathrm{t}=0.29$ & 108 & $.722 \mathrm{NS}$ \\
\hline \multirow[t]{6}{*}{ Profesión } & Ama de casa & $35.5 \%(39)$ & $36.0 \%(18)$ & $35.0 \%(21)$ & & & \\
\hline & Obrero & $33.6 \%(37)$ & $38.0 \%(19)$ & $30.0 \%(18)$ & & & \\
\hline & $\begin{array}{l}\text { Empleado } \\
\text { cualificado }\end{array}$ & $12.7 \%(14)$ & $10.0 \%(5)$ & $15.0 \%(9)$ & Chi2 $=5.61$ & 4 & $.231 \mathrm{NS}$ \\
\hline & Autónomo & $13.6 \%(15)$ & $8.0 \%$ & $18.3 \%(11)$ & & & \\
\hline & $\begin{array}{l}\text { Profesional } \\
\text { especialista }\end{array}$ & $4.5 \%(5)$ & $8.0 \%$ & $1.7 \%(1)$ & & & \\
\hline & Diestra & $95.5 \%(105)$ & $96.0 \%(48)$ & $95.0 \%(57)$ & & & \\
\hline \multirow[t]{2}{*}{ Lateralidad } & Zurda & $2.7 \%(3)$ & $2.0 \%(1)$ & $3.3 \%(2)$ & Chi $2=0.20$ & 2 & $.906 \mathrm{NS}$ \\
\hline & Ambidiestra & $1.9 \%(2)$ & $2.0 \%$ & $1.7 \%(1)$ & & & \\
\hline
\end{tabular}

N.S. $=$ NO significativo $(\mathrm{P}>.05) \quad * *=$ Altamente significativo al $1 \%(\mathrm{P}<.01)$.

Abreviaciones: DTA, Demencia tipo Alzheimer; Estud, Estudios; Gl, Grado de libertad, N, muestra; P, p-value sig.; SSDC, Sujetos sin deterioro cognitivo

En la Tabla 2 aparecen los resultados obtenidos en la CV. Los sujetos con DTA tienen una media menor de $\mathrm{CV}$ global auto percibida que los SSDC siendo la diferencia significativa $(\mathrm{t}=-4.76 ; \mathrm{P}<.001)$ con un tamaño del efecto moderado (.186).

Tabla 2: Test de diferencia entre 2 medias. Calidad de vida global auto percibida en función del Grupo.

\begin{tabular}{|c|c|c|c|c|c|c|c|c|c|c|c|}
\hline \multirow{2}{*}{ Variable / } & \multirow{2}{*}{ Group } & \multirow{2}{*}{$\mathrm{N}$} & \multirow{2}{*}{ Media (IC al 95\%) } & \multirow{2}{*}{ D.E. } & \multicolumn{3}{|c|}{ T Student } & \multirow{2}{*}{$\begin{array}{l}\text { IC 95\% dif } \\
\text { absoluta }\end{array}$} & \multicolumn{2}{|c|}{ Test Mann-W } & \multirow{2}{*}{$\begin{array}{l}\text { Tamaño } \\
\text { del } \\
\text { efecto: } \\
\text { R2 }\end{array}$} \\
\hline & & & & & $\mathrm{T}$ & $\mathrm{Gl}$ & $\mathrm{P}$ & & /Z/ & $\mathrm{P}$ & \\
\hline \multirow[t]{2}{*}{ QOL-AD P. Total } & DTA & 50 & $34.38(33.29-35.47)$ & 3.83 & \multirow{2}{*}{-4.76} & \multirow{2}{*}{108} & \multirow{2}{*}{$.000 *$} & \multirow{2}{*}{$1.72-41.18$} & \multirow{2}{*}{5.04} & \multirow{2}{*}{$.000 *$} & \multirow{2}{*}{.186} \\
\hline & SSDC & 60 & $37.33(36.73-37.94)$ & 2.33 & & & & & & & \\
\hline
\end{tabular}

$$
* *=\text { Altamente significativo al } 1 \%(\mathrm{P}<.01)
$$

\section{Discusión}

La hipótesis principal se basa en que los pacientes con DTA presentan una peor percepción de la CV global auto percibida comparando los resultados con los obtenidos por SSDC con características sociodemográficas similares. En los resultados obtenidos se confirman la hipótesis sobre la percepción de la CV global, de modo que los sujetos con DTA obtienen resultados inferiores que los SSDC. Otros estudios que han comparado la percepción de la CV de los pacientes con sus cuidadores principales han demostrado que los pacientes presentan una percepción más positiva sobre su CV. Las posibles discrepancias entre la percepción de ambos, 
puede estar basada en el nivel de conciencia del paciente así como en la carga que sufre el cuidador principal (Sousa, Santos, Simões, Conde-Sala, Dourado, 2017).

Tras realizar una validación de la escala QoL-AD en pacientes y profesionales sanitarios se demostró que no existía diferencia entre los pacientes más afectados cognitivamente o con menor nivel de conciencia en los índices de consistencia interna obtenidos en la escala QoL-AD (Gómez-Gallego, Gómez-Amor, Gómez- García, 2012). Trabajos recientes realizados por Conde-Sala et al., (2016), mediante un estudio longitudinal con 127 participantes durante 24 meses con el objetivo de analizar los parámetros que más se relacionan con la autopercepción en la $\mathrm{CV}$, demostraron que los factores vinculados con la depresión, una menor habilidad funcional y la presencia de anosognosia eran predictivos de la menor autopercepción de la CV. Otros estudios han evidenciado que los pacientes que tienen mayor anosognosia, es decir, menor nivel de conciencia sobre la propia enfermedad y su deterioro cognitivo, muestran puntuaciones más altas en la CV auto percibida (Portellano-Ortiz et al, 2014).

Por tanto, estos hallazgos hay que tenerlos en consideración por sus implicaciones clínicas, ya que a mayor gravedad de la demencia, mayor deterioro cognitivo y mayor anosognosia, conllevaría, según los resultados anteriores, una mejor percepción de la CV. Esta sería como consecuencia de una sobrevaloración producida por la falta de conciencia sobre los propios déficits. Por esta razón, una limitación del estudio consiste en la ausencia de valoración de la conciencia del déficit, ya que puede suponer una variable extraña que puede condicionar los resultados obtenidos. De manera similar, la ausencia de análisis de la influencia de la variable edad, ya que puede distorsionar los resultados obtenidos entre las variables objeto de estudio. Otra limitación consiste en la utilización de una única medida de evaluación de la CV. Como posibles líneas de investigación sería la realización de estudios longitudinales con pacientes con DTA en diferentes niveles de deterioro cognitivo, teniendo en cuenta el nivel de conciencia y la utilización de diferentes medidas específicas que valoren la $\mathrm{CV}$.

\section{Conclusión}

La calidad de vida global auto percibida es menor en los pacientes con demencia tipo Alzheimer que en los sujetos sin deterioro cognitivo.

Se confirma la hipótesis de que a mayor deterioro cognitivo mayor afectación en la calidad de vida.

\section{Bibliografía}

American Psychiatric Association, A. P. A. (2002). Diagnostic and statistical manual of mental disorder (fourth edition ed.). Washington, DC: APA.

Bosboom, P.R., Alfonso, H., Almeida, O.P. (2013). Determining the predictors of change in quality of life self-ratings and carer-ratings for community-dwelling people with Alzheimer disease. Alzheimer Dis Assoc Disord, 27(4), 363-71. 
Conde-Sala, J.L., Reñé-Ramírez, R., Turró-Garriga, O., Gascón-Bayarri, J., CampdelacreuFumadó, J., Juncadella-Puig, M., Rico-Pons, I., Garre-Olmo, J. (2014). Severity of dementia, anosognosia, and depression in relation to the quality of life of patients with Alzheimer disease: discrepancies between patients and caregivers. Am J Geriatr Psychiatry. 22(2), 138-47.

Conde-Sala, J.L., Turró-Garriga, O., Portellano-Ortiz, C., Viñas-Diez, V., Gascón-Bayarri, J., Reñé-Ramírez, R. (2016). Self-Perceived Quality of Life Among Patients with Alzheimer's Disease: Two Longitudinal Models of Analysis. Journal of Alzheimer's Disease 52, 9991012.

Gómez-Gallego, M. G. (2012). Estudio del acuerdo entre las valoraciones de la calidad de vida de pacientes con la enfermedad de alzheimer y sus cuidadores. Alzheimer. Real Invest Demenc, 15, 22-27.

Gomez-Gallego, M., Gòmez-Amor, J., Gòmez- Garcìa, J., 2012.Validación de la versión española de la escala QoL-AD en pacientes con enfermedad de Alzheimer, cuidadores y profesionales sanitarios. Neurología, 27(1):4-10.

Hoe, J., Hancock, G., Livingston, G., Woods, B., Challis, D., y Orrell, M. (2009). Changes in the quality of life of people with dementia living in care homes. Alzheimer Disease and Associated Disorders, 23(3), 285-290. doi: 10.1097/WAD.0b013e318194fc1e.

Lobo, A., Saz, P., Marcos, G., y ZARADEMP, g. (2002). Examen Cognoscitivo. Madrid: TEA Eds.

Logsdon, R. G., Gibbons, L. E., McCurry, S. M., y Teri, L. (1999). Quality of life in Alzheimer's disease: Patient and caregiver reports. Journal of Mental Health \& Aging, 5(1), 21-32.

McKhann, Drachman, D., Folstein, M., Katzman, R., Price, D., y Stadlan, E. M. (1984). Clinical diagnosis of Alzheimer's disease: report of the NINCDS-ADRDA Work Group under the auspices of Department of Health and Human Services Task Force on Alzheimer's Disease. Neurology, 34(7), 939-944.

Nogueira, M. N. Simões, J.P. de Sousa, M.F., Santos, R.L., Rosa, R., Belfort, T., Torres, B., Dourado, M.C.N. (2015). Spouse-caregivers' quality of life in Alzheimer's disease. Int Psychogeriatr. 27(5), 837-45.

Perea, M. V. (2005). Demencia. En I. M. (Ed.), Síndromes neuropsicologicos (págs. 175-196). Salamanca: Amarú Ediciones.

Portellano-Ortiz, C., Turró-Garriga, O., Gascón-Bayarri, J., Piñán-Hernández, S., MorenoCordón, L., Viñas-Díez, V., Reñé-Ramírez, R., Conde-Sala, J.L. (2014). Anosognosia y depresión en la percepción de la calidad de los pacientes con enfermedad de Alzheimer. Rev Neurol, 59 (5) , 193-204. 
Sousa, M.F.B., Santos, R.L., Simões, J.P,, Conde-Sala, J.L., Dourado, M.C.N. (2017). Discrepancies Between Alzheimer's Disease Patients' and Caregivers' Ratings About Patients' Quality of Life: A 1-year Observation Study in Brazil. Alzheimer Dis Assoc Disord. .

WHO. (1994). Quality of life Assessment. An annotated Bibliography. Division. Geneva: World Health Organization. 